\title{
The linear growth of structure in the $R_{\mathrm{h}}=c t$ universe
}

\author{
Fulvio Melia ${ }^{\star} \dagger$ \\ Department of Physics, The Applied Math Program, and Department of Astronomy, The University of Arizona, AZ 85721, USA
}

Accepted 2016 September 29. Received 2016 September 27; in original form 2016 June 24; Editorial Decision 2016 September 27

\begin{abstract}
We use recently published redshift space distortion measurements of the cosmological growth rate, $f \sigma_{8}(z)$, to examine whether the linear evolution of perturbations in the $R_{\mathrm{h}}=c t$ cosmology is consistent with the observed development of large-scale structure. We find that these observations favour $R_{\mathrm{h}}=c t$ over the version of $\Lambda$ cold dark matter $(\Lambda \mathrm{CDM})$ optimized with the joint analysis of Planck and linear growth rate data, particularly in the redshift range $0<$ $z<1$, where a significant curvature in the functional form of $f \sigma_{8}(z)$ predicted by the standard model — but not by $R_{\mathrm{h}}=c t$-is absent in the data. When $\Lambda$ CDM is optimized using solely the growth rate measurements; however, the two models fit the observations equally well though, in this case, the low-redshift measurements find a lower value for the fluctuation amplitude than is expected in Planck $\Lambda \mathrm{CDM}$. Our results strongly affirm the need for more precise measurements of $f \sigma_{8}(z)$ at all redshifts, but especially at $z<1$.
\end{abstract}

Key words: gravitation - instabilities - cosmological parameters - cosmology: observations cosmology: theory - large-scale structure of Universe.

\section{INTRODUCTION}

The large-scale structure revealed by the distribution of galaxies is believed to have formed through a process of gravitational instability, starting with primordial fluctuations in the early Universe. But while self-gravity amplifies perturbations in the cosmic fluid, cosmic expansion suppresses them. Their growth rate therefore depends rather sensitively on the dynamical expansion of the Universe and may be used to discriminate between different models. Measurements of the growth rate tend to focus on infall motions associated with condensing regions, with peculiar velocities largely correlated with the local gravitational potential. Galaxies trace these motions, carrying an imprint of the changing growth rate as the Universe evolves (Peacock et al. 2001; Viel, Haehnelt \& Springel 2004; Jain \& Zhang 2007; Ross et al. 2007; da Angela et al. 2008; Guzzo et al. 2008; Song \& Koyama 2009; Song \& Percival 2009; Davis et al. 2011; Hudson \& Turnbull 2012; Macaulay, Wehus \& Eriksen 2013; Alam, Ho \& Silvestri 2016).

A principal statistical technique used to measure the growth rate is based on the redshift space distortion (RSD) created by the galaxies' peculiar velocities (Kaiser 1987). Specifically, maps produced with distances inferred from redshifts in spectroscopic galaxy surveys show that the galaxy distributions are anisotropic due to the fact that the redshifts contain components from both the smooth Hubble flow and the peculiar velocities of the infalling matter. As long as one can reliably separate these two contributions to the redshift, one may thereby extract a history of the build-up of structure.

^E-mail: fmelia@email.arizona.edu

$\dagger$ John Woodruff Simpson Fellow.
The use of RSD, however, is practical primarily before nonlinear effects begin to emerge, where measurements yield information on both the matter overdensity and the peculiar velocities of galaxies. In the linear regime, the problem is typically reduced to solving a second-order differential equation for the time-dependent fluctuations, from which one may then infer their growth rate. Thus, although objects suitable for this work can in principle include individual galaxies, clusters of galaxies, and superclusters, their density contrasts $(\delta \rho / \rho)$ today are, respectively, $\sim 10^{6}, \sim 10^{3}$, and $\sim O(1)$. These estimates assume a critical density $\rho_{\mathrm{c}} \equiv 3 c^{2} H_{0}^{2} / 8 \pi G \sim 10^{-29} \mathrm{~g} \mathrm{~cm}^{-3}$, and that about $10^{12} \mathrm{M}_{\odot}$ of galactic matter are contained within an $\sim 30 \mathrm{kpc}$ region. Clusters typically contain fewer than $10^{3}$ galaxies, while superclusters have tens of thousands of galaxies. In $\Lambda$ cold dark matter $(\Lambda \mathrm{CDM}), \rho \sim$ $(1+z)^{3}$ during the matter-dominated era, so galaxies and clusters presumably ceased growing linearly, i.e. grew with $\delta \rho<1$, at redshifts $\sim 100$ and $\sim 10$, respectively. These structures are non-linear in the local neighbourhood. The linear-growth analysis described in this paper therefore tends to address the formation of superclusters, which could have grown linearly over the redshift range $z<2-3$. This is the approach we shall follow in this paper, and therefore focus on surveys relevant to these large structures, including 2dFGRS (Peacock et al. 2001) and VVDS (Guzzo et al. 2008).

By now, the development of linear perturbation theory is quite mature - at least within the context of the standard model $(\Lambda \mathrm{CDM})$, in which dark energy corresponds to a cosmological constant $\Lambda$. Comparative tests using the growth of linear structure have been carried out between $\Lambda \mathrm{CDM}$ and an assortment of other cosmologies, principally those based on extensions to general relativity (GR) involving higher order curvature terms or extra dimensions (Wetterich 1995; Amendola 2000; Dvali, Gabadadze \& 
Porrati 2000; Carroll et al. 2004; Capozziello, Cardone \& Troisi 2005). However, measurements of the linear growth rate have not yet been used to test the $R_{\mathrm{h}}=c t$ universe (Melia 2007; Melia \& Shevchuk 2012; Melia 2016a, 2017), another FriedmannRobertson-Walker (FRW) cosmology, which has thus far been shown to fit many other kinds of data better than $\Lambda$ CDM. (A brief summary of these previously published results is provided in Section 3.1.) The principal aim of this paper is to address this deficiency.

We are especially motivated to carry out this analysis by recent comparative studies using the Alcock-Paczyński test (Alcock \& Paczyński 1979), based on the changing ratio of angular to spatial/redshift size of (presumed) spherically symmetric source distributions with distance (Melia \& López-Corredoira 2016). The use of this diagnostic, with newly acquired measurements of the anisotropic distribution of baryon acoustic oscillation (BAO) peaks from the Baryon Oscillation Spectroscopic Survey of the Sloan Digital Sky Survey III release (SDSS III/BOSS DR11) at average redshifts $\langle z\rangle=0.57$ and $\langle z\rangle=2.34$, has allowed us to determine the geometry of the Universe with unprecedented accuracy. Previous applications of the galaxy two-point correlation function to measure a redshift-dependent scale that could be used to determine the ratio of angular (i.e. transverse) size to redshift (i.e. radial) size were limited by the need to disentangle the acoustic scale in redshift space from redshift distortions from internal gravitational effects (López-Corredoira 2014). A major limitation of this process was that inevitably one had to pre-assume a particular cosmological model, or adopt prior parameter values, in order to estimate the possible confusion between the true cosmological redshift interval from one edge of the cluster to the other and the contribution to this redshift width from these internal gravitational effects. Unfortunately, the wide range of possible distortions for the same correlation-function shape resulted in very large errors associated with the BAO peak position and hence the inferred acoustic scale (often in the $\sim 20-30$ per cent range).

This situation has improved significantly over the past few years with (1) the use of reconstruction techniques (Eisenstein et al. 2007; Padmanabhan et al. 2012) that enhance the quality of the galaxy two-point correlation function, and (2) the use of Ly $\alpha$ and quasar observations to more precisely determine their auto- and cross-correlation functions, allowing the measurement of BAO peak positions to better than $\sim 4$ percent accuracy (Cuesta et al. 2016). The most recent determination of $y(z)$ has been based on the use of three BAO peak positions: the measurement of the BAO peak position in the anisotropic distribution of SDSS-III/BOSS DR12 galaxies at $\langle z\rangle=0.32$ and $\langle z\rangle=0.57$ (Cuesta et al. 2016), in which a technique of reconstruction to improve the signal/noise ratio was applied; and the self-correlation of the BAO peak in the Ly $\alpha$ forest in the SDSS-III/BOSS DR11 data at $\langle z\rangle=2.34$ (Padmanabhan et al. 2012), plus the cross-correlation of the BAO peak of quasi-stellar objects (QSOs) and the Ly $\alpha$ forest in the same survey (Font-Ribera et al. 2014).

With these new measurements, the use of the Alcock-Paczyński diagnostic (Melia \& López-Corredoira 2016) has shown that the current concordance $(\Lambda \mathrm{CDM})$ model is disfavoured by the BAO data at $2.6 \sigma$. They instead show that the $R_{\mathrm{h}}=c t$ model has a probability $\sim 0.68$ (i.e. consistent with 1 ) of being correct. Measurements of the linear growth rate also critically depend on the Alcock-Paczyński effect, so the observations considered in this paper provide an invaluable, complementary, set of data with which to test the $R_{\mathrm{h}}=c t$ cosmology.

In Section 2 of this paper, we derive the necessary formalism for studying the time evolution of linear fluctuations in this model, which reduces to solving a second-order differential equation, though with some important differences compared with its counterpart in $\Lambda$ CDM. A contextual background for $R_{\mathrm{h}}=c t$ is provided in Section 3, where we also solve the growth equation as a function of redshift, and describe the observables, specifically the volume-delimited variance $\sigma_{8}(z)$ of the fluctuations and its corresponding growth function. The standard model is analysed in Section 4, and we end with a discussion and conclusion in Sections 5 and 6.

\section{RELATIVISTIC PERTURBATION THEORY}

The equations describing the growth of linear perturbations in $\Lambda \mathrm{CDM}$ are well known so, for this model, we will simply adopt the key results from previous work and summarize these in Section 4 below. In the case of $R_{\mathrm{h}}=c t$, however, it is essential to begin with relativistic perturbation theory, which we now describe. We will assume that small inhomogeneities emerge into the semiclassical universe out of the Planck regime (Melia 2016b) with an essentially scale-free distribution (see below). The subsequent development of structure is usually assumed to have progressed through a series of steps, each corresponding to a particular, dominant component in the cosmic fluid, eventually leading to the formation of stars and galaxies. To study the growth of perturbations in an otherwise smooth background, it is helpful to know (i) the relative abundance of non-relativistic baryonic (b) and cold dark matter $(\mathrm{cdm})$; (ii) the contribution to the total energy density $\rho$ and pressure $p$ from all the components in the cosmic fluid; and (iii) the spectrum and type (i.e. adiabatic or isothermal) of primeval density fluctuations.

Newtonian theory, as a limiting approximation to GR, is only applicable within the Hubble radius $R_{\mathrm{h}} \equiv c / H(t)$, where the effects of space-time curvature are small. In the $R_{\mathrm{h}}=c t$ universe, the quantum fluctuations always have a wavelength (or scale) $\lambda$ smaller than $R_{\mathrm{h}}$, and the perturbations that grow from these early seeds never stretch in size beyond this gravitational horizon. But the full GR theory is none the less still necessary when we are dealing with perturbations in relativistic matter, such as would occur in a baryonic fluid coupled to the radiation field, or in a fluid that includes energetic neutrinos.

We consider perturbations about the background solution for the density and pressure, labelled with subscript 0 , such that

$\rho=\rho_{0}+\delta \rho$,

and

$p=p_{0}+\delta p$,

where $\delta \rho$ and $\delta p$ are the perturbed first-order variables that generally depend on the spatial coordinates $x^{i}$ as well as the time $\left(x^{0}=c t\right)$. (In this paper, we denote the spatial coordinates with indices $i, j$, $k$, while Greek indices refer to all four coordinates, with metric signature $[+,-,-,-]$.) In the linear regime, we have $|\delta \rho| \ll$ $\rho$, and similarly for all the other first-order quantities. Different perturbative modes therefore evolve independently of each other and may be treated separately.

For simplicity, we consider only barotropic fluids with $p=p(\rho)$ and, following convention, we use a dimensionless density variable $\delta \equiv \delta \rho / \rho_{0}$, etc. in all the equations. We define the background equation-of-state parameter

$w \equiv \frac{p_{0}}{\rho_{0}}$ 
which includes the contributions to $\rho_{0}$ and $p_{0}$ from all the components in the cosmic fluid, and the adiabatic sound speed, $v_{\mathrm{s}}$, within the fluctuation, where $\partial_{\alpha} \delta p=v_{\mathrm{s}}^{2} \partial_{\alpha} \delta \rho$, so that

$v_{\mathrm{s}}^{2} \equiv \frac{\mathrm{d} p}{\mathrm{~d} \rho}$,

which depends principally on the perturbed quantities $\delta p$ and $\delta \rho$.

In deriving the dynamical equations for the evolution of the perturbations, one must carefully distinguish between the different roles played by the background quantities $\rho_{0}$ and $p_{0}$, and the fluctuations $\delta \rho$ and $\delta p$. The former dominate the evolution of the Hubble constant $H(t)$ in (cosmic) time $t$, while the local growth is heavily influenced by the gravitational potential associated with the fluctuations themselves. Of course, the Einstein field equations contain a single stress-energy tensor $\boldsymbol{T}^{\mu v}$ encompassing all of the sources (i.e. $\left.\rho=\rho_{0}+\delta \rho, p=p_{0}+\delta p\right)$ but, as we shall see, once the dynamical equations are linearized, some terms depend predominantly on $\rho_{0}$ and $p_{0}$, while others contain only the perturbed amplitudes. The equations we will use allow for the possible evolution of a matter perturbation embedded within an otherwise smooth background, such as we would encounter in a radiation-dominated, or darkenergy-dominated, universe. In every case, however, the expansion rate is always driven by $\rho \approx \rho_{0}$ and $p \approx p_{0}$.

In the relativistic treatment of fluctuation growth, we perturb both the space-time metric and the stress-energy tensor $\boldsymbol{T}^{\mu \nu}$ representing the sources. The details of how one linearizes the Einstein field equations have appeared in many previous publications, and we refer the reader to some of these excellent works (Weinberg 1972; Landau \& Lifshitz 1975; Peebles 1980, 1993; Press \& Vishniac 1980; Kolb \& Turner 1990; Padmanabhan 1993; Coles \& Lucchin 1995; Peacock 1999; Liddle \& Lyth 2000; Tsagas, Challinor \& Maartens 2008). We follow the covariant Lagrangian approach, employing locally defined quantities, and we derive their evolution along the worldliness of comoving observers. There is therefore a slight difference between the proper time $\tau$ defined at each spacetime point, and the cosmic time $t$, since the perturbations somewhat shift the local frame out of the Hubble flow (in which $t$ would otherwise be the proper time everywhere). Fortunately, there is a straightforward way to deal with such 'gauge' issues using the coordinate transformation

$\frac{\mathrm{d} \tau}{\mathrm{d} t}=1-\frac{\delta p}{\rho+p}$.

The energy conservation law reads

$\frac{\mathrm{d} \rho}{\mathrm{d} \tau}=-3 H(\rho+p)$,

and it is straightforward to see from equations (5) and (6) that (Padmanabhan 1993; Liddle \& Lyth 2000)

$\frac{\mathrm{d}(\delta \rho)}{\mathrm{d} t}=-3 H_{0} \delta \rho-3 \delta H\left(\rho_{0}+p_{0}\right)$.

In addition,

$\frac{\mathrm{d}}{\mathrm{d} t}(\delta H)+2 H_{0} \delta H+\frac{4 \pi G}{3 c^{2}} \rho_{0} \delta+\frac{v_{\mathrm{s}}^{2}}{3(1+w)} D^{2} \delta=0$,

and

$\dot{H}_{0}=-\frac{3}{2}(1+w) H_{0}^{2}$,

where $H(t)=H_{0}(t)+\delta H(t), H_{0}(t)$ is the smoothed Hubble constant driven by the background fluid (i.e. $\rho_{0}$ and $p_{0}$ ) at time $t, \delta H(t)$ describes scalar deviations from the smooth background expansion rate represented by $H_{0}$, and $D^{2}$ is the four-dimensional Laplacian operator. $\left(H_{0}\right.$ should not be confused with the Hubble constant today, which is usually also denoted with subscript ' 0 '.)

As we discuss below, $\rho_{0}$ in the $R_{\mathrm{h}}=c t$ universe appears to be dominated by dark energy and (baryonic and dark) matter at low redshifts, and dark energy and radiation in the early Universe. In this paper, we focus on the more recent growth of perturbations, and we follow the conventional approach of assuming that dark energy $\left(\rho_{\mathrm{de}}\right)$ is a smooth background, while the perturbations themselves are due solely to fluctuations in the matter density $\rho_{\mathrm{m}}$. We further assume, again conventionally, that once these matter perturbations start to condense out of the smooth cosmic fluid, they decouple from the dark energy, except through their gravitational interaction.

We shall see shortly that in the $R_{\mathrm{h}}=c t$ universe, where $\rho+3 p=$ $0, \delta$ is constant to first order at low redshifts. In solving equations (7)-(9), we will therefore also retain the leading second-order terms to determine its time dependence, if any. From equation (7), it is straightforward to show that

$\dot{\delta}=-H_{0} \delta-2 \delta H-3 \delta H \delta$,

and therefore from equations (8)-(10), we find that

$\ddot{\delta}+3 H_{0} \dot{\delta}-\frac{3}{2}\left(\dot{\delta}^{2}-H_{0} \delta \dot{\delta}+H_{0}^{2} \delta^{2}\right)=v_{\mathrm{s}}^{2} D^{2} \delta$.

To first order, the gravitational (third) term on the left-hand side is zero, a result of the zero active mass condition $\rho+3 p=0$, for which the cosmic fluid experiences no net gravitational acceleration. We therefore assume that $\delta$ is (at most) a very weak function of $t$,

$\delta(t) \sim t^{\alpha}$,

with $|\alpha| \ll 1$. In that case,

$\frac{3}{2}\left(\dot{\delta}^{2}-H_{0} \delta \dot{\delta}+H_{0}^{2} \delta^{2}\right)=\frac{1}{t^{2}}\left(\alpha^{2} \delta^{2}-\alpha \delta^{2}+\delta^{2}\right)$,

which we approximate as

$\frac{3}{2}\left(\dot{\delta}^{2}-H_{0} \delta \dot{\delta}+H_{0}^{2} \delta^{2}\right) \approx A t^{-2} \delta$,

where $A$ is essentially constant with $|A| \ll 1$. And so equation (11) reduces to the form

$\ddot{\delta}+3 H_{0} \dot{\delta}-\frac{A}{t^{2}} \delta=v_{\mathrm{s}}^{2} D^{2} \delta$.

The derivative term in equation (15) requires some knowledge concerning the spatial variation of the fluctuation. This is typically handled via a wavenumber decomposition of $\delta \rho$, in which the density fluctuation is written as a Fourier series,

$\delta\left(x^{\alpha}\right)=\frac{1}{(2 \pi)^{3}} \int \tilde{\delta}_{k}(t) \mathrm{e}^{-\mathrm{i} k \cdot x} d^{3} k$,

in terms of the comoving wavenumber $k$ and wavevector $k^{\alpha} \equiv$ $\left(\omega_{k} / c, \boldsymbol{k}\right)$ where, as always, $k=|\boldsymbol{k}|$. The Fourier components may be calculated through the expression

$\tilde{\delta}_{k}(t)=\int \delta\left(x^{\alpha}\right) \mathrm{e}^{\mathrm{i} k \cdot x} \mathrm{~d}^{3} x$.

With this, and the fact that $H_{0}=1 / t$, it is straightforward to show from equation (15) that the local growth rate equation in the $R_{\mathrm{h}}=$ $c t$ universe may be written as

$\frac{\mathrm{d}^{2} \tilde{\delta}_{k}}{\mathrm{~d} t^{2}}+\frac{3}{t} \frac{\mathrm{d} \tilde{\delta}_{k}}{\mathrm{~d} t}-\frac{A}{t^{2}} \tilde{\delta}_{k}=-\frac{k^{2}}{a^{2}} v_{\mathrm{s}}^{2} \tilde{\delta}_{k}$,

where $a(t)$ is the universal expansion factor. Note that for the application we are considering in this paper, we have explicitly used 
the constant value $w-1 / 3$, so terms proportional to $\dot{w}$ have been omitted from these expressions (cf. Tsagas et al. 2008).

These equations specifically describe the evolution of scalar fluctuations. It is well known that the inclusion of metric perturbations about the spatially flat FRW background metric produces a combination of modes that conveniently split into scalar, vector, and tensor components, depending on how they transform on spatial hypersurfaces. But vector perturbations have no lasting influence in an expanding universe. In addition, scalar and tensor modes decouple to linear order, so gravity waves do not provide any backreaction to the metric; they satisfy sourceless equations when the energymomentum tensor is diagonal, as is usually assumed for a perfect fluid in cosmology. For these reasons, we focus exclusively on the evolution of scalar perturbations in this paper.

\section{PERTURBATION GROWTH IN THE $\boldsymbol{R}_{\mathrm{h}}=c t$ UNIVERSE}

\subsection{Background}

Let us first briefly discuss the motivation for considering this model. We have been developing this FRW cosmology for over 10 years now (Melia 2007, 2016a, 2017; Melia \& Shevchuk 2012), driven largely by a series of observational tests that suggest its predicted expansion rate is a better fit to the data than that of the current concordance model, $\Lambda \mathrm{CDM}$. In this subsection, we survey some of these completed model comparisons, and explain why it is now necessary to probe this cosmology more deeply, including its predicted growth rate-the subject of this paper.

Since competing models tend to have different formulations, often with unmatched parameters, one must use model selection tools to determine which (if any) is preferred by the data. It is now common in cosmology to use tools such as the Akaike information criterion (AIC; Liddle 2007), the Kullback information criterion (KIC; Cavanaugh 2004), and the Bayes information criterion (BIC; Schwarz 1978) for this purpose. When using the AIC, with $\mathrm{AIC}_{\alpha}=-2 \ln \mathcal{L}_{\alpha}+2 n_{\alpha}$ characterizing model $\mathcal{M}_{\alpha}$, the difference $\Delta_{\mathrm{AIC}}=\mathrm{AIC}_{2}-\mathrm{AIC}_{1}$ determines the extent to which model $\mathcal{M}_{1}$ is favoured over model $\mathcal{M}_{2}$. Here, $\mathcal{L}$ is the maximum value of the likelihood function and $n$ is the number of free parameters (see Melia \& Maier 2013, and references cited therein, for more details). For Kullback and Bayes, the likelihoods are defined analogously. In using these model selection tools, the outcome $\Delta$ (for AIC, KIC, or $\mathrm{BIC}$, as the case may be) is judged to represent 'positive' evidence that model 1 is preferred over model 2 if $\Delta>2$. If $2<\Delta<6$, the evidence favouring model 1 is moderate, and it is very strong when $\Delta>10$. Sometimes, the outcome $\Delta$ is used to estimate the relative probability (or percentage likelihood) that $\mathcal{M}_{1}$ is statistically preferred over $\mathcal{M}_{2}$, according to the prescription

$P\left(\mathcal{M}_{1}\right)=\frac{1}{1+\exp (-\Delta / 2)}$,

with $P\left(\mathcal{M}_{2}\right)=1-P\left(\mathcal{M}_{1}\right)$, when only two models are being compared directly.

In Table 1 , we quote the outcome $\Delta \equiv \Delta_{\Lambda \mathrm{CDM}}-\Delta_{R_{\mathrm{h}}=c t}$, or the percentage likelihoods calculated from it, for $R_{\mathrm{h}}=c t$ and $\Lambda \mathrm{CDM}$, based on the 18 tests published thus far. All of these outcomes have consistently favoured $R_{\mathrm{h}}=c t$ over $\Lambda \mathrm{CDM}$, sometimes moderately, often very strongly. Yet in spite of these consistently favourable comparisons, some have been critical of the $R_{\mathrm{h}}=c t$ model. For example, in contrast to the conclusions regarding the SNLS Type Ia SNe by Wei et al. (2015a), Shafer (2015) compared cosmo- logical models using both the Union2.1 (Suzuki et al. 2012) and JLA (Betoule et al. 2014) SN samples and argued that $\Lambda \mathrm{CDM}$ was strongly favoured by these data. However, he appears to have incorrectly estimated the intrinsic dispersion of each subsample, and additionally failed to include them in his maximum likelihood estimation, which greatly biased his analysis. Shafer (2015) also analysed measurements of $H(z)$ versus $z$, but here too he avoided using truly model-independent cosmic chronometer measurements, opting instead to use heavily biased BAO estimates. He appears to have been unaware of the significant limitations of all but the most recent 2 or 3 BAO scale determinations. A similar study, also based on model-dependent estimates of $H(z)$ versus $z$, was carried out by Bilicki \& Seikel (2012). In contrast, when one uses truly model-independent cosmic chronometer observations, the outcome strongly favours $R_{\mathrm{h}}=c t$ over $\Lambda$ CDM (see e.g. Melia \& Maier 2013; Melia \& McClintock 2015a).

Taking a different approach, van Oirschot, Kwan \& Lewis (2010), and Lewis (2013) argued that the definition of the gravitational horizon $R_{\mathrm{h}}$ in the $R_{\mathrm{h}}=c t$ universe is ill-defined and that light rays emitted from beyond it are none the less still detectable. But their analysis was flawed because it was based on an improper use of null geodesics in the FRW metric. A full accounting of this, and a proof that no null geodesics reaching us today originated from beyond $R_{\mathrm{h}}$, appeared in Bikwa, Melia \& Shevchuk (2012) and Melia (2012).

And in two of the more recent claims made against $R_{\mathrm{h}}=c t$, Mitra (2014) has argued that this cosmology is static and merely represents another vacuum solution, while Lewis (2013) attempted to show that the equation of state in $R_{\mathrm{h}}=c t$ is inconsistent with $p=-\rho / 3$, thereby ruining the elegant, high-quality fits to the observations. As shown in Melia (2015c), however, these criticisms are based on either incorrect assumptions or basic theoretical errors. For example, Florides (1980) proved in his landmark paper that there are six - and only six - special cases of the FRW metric for which one may transform the coordinates into a frame where the metric coefficients are static. The $R_{\mathrm{h}}=c t$ model is not one of them. Mitra (2014) erroneously concluded that this cosmology has a constant expansion rate because its density is zero. In fact, the linear expansion occurs because $R_{\mathrm{h}}=c t$ has zero active mass, i.e. $\rho+3 p=0$, not because $\rho=0$. Lewis's (2013) analysis was even more superficial than this, because he based his conclusion on the inexplicable assumption that $\rho$ must have only a single component in the $R_{\mathrm{h}}=c t$ universe. Like $\Lambda \mathrm{CDM}$, however, $\rho$ in this cosmology has multiple components, including radiation, matter, and the poorly known dark energy. But these two models differ in the critical constraint that the total $p$ must always be equal to $-\rho / 3$ in $R_{\mathrm{h}}=c t$, though not in $\Lambda \mathrm{CDM}$. A more detailed explanation of how these components vary with redshift may be found in Melia \& Fatuzzo (2016).

The growth rate described in this paper addresses one of the few remaining areas where an examination of how well these two models account for the data has yet to be made. As we shall see, our results indicate that the measured growth rate favours $R_{\mathrm{h}}=c t$ over $\Lambda \mathrm{CDM}$, in complete agreement with the outcomes presented in Table 1 .

\subsection{Growth equation in the $\boldsymbol{R}_{\mathrm{h}}=c t$ universe}

Let us now apply the growth equation to the $R_{\mathrm{h}}=c t$ universe, in which (like $\Lambda \mathrm{CDM}$ ) the energy density contains at least five components: (1) cold dark matter (cdm), (2) baryonic matter (b), (3) photons $(\gamma)$, (4) neutrinos $(v)$, and (5) dark energy (de), so that generally

$\rho=\rho_{\mathrm{m}}+\rho_{\mathrm{r}}+\rho_{\mathrm{de}}$. 


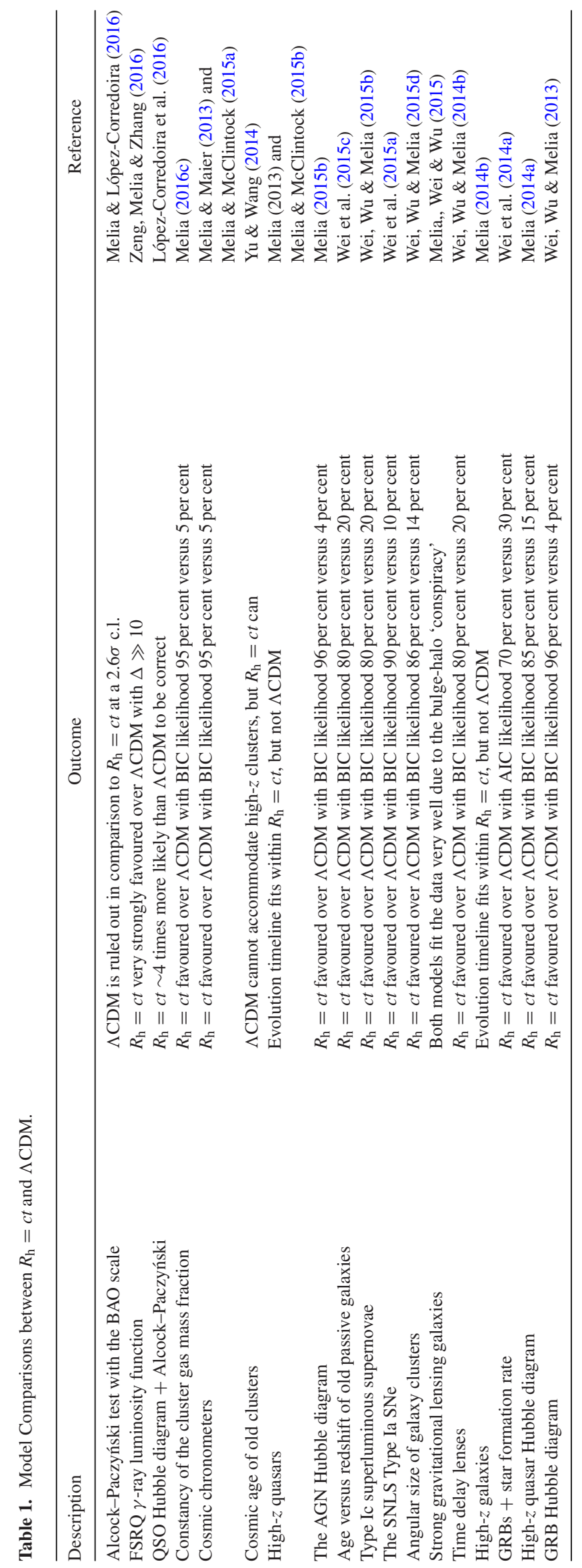


The baryons and cold dark matter are often grouped together,

$\rho_{\mathrm{m}}=\rho_{\mathrm{b}}+\rho_{\mathrm{cdm}}$,

as are the photons and neutrinos when the latter are still relativistic:

$\rho_{\mathrm{r}}=\rho_{\gamma}+\rho_{\nu}$.

At least within the standard model $(\Lambda \mathrm{CDM})$, baryons and photons interact with each other up until the time of decoupling, $t_{\mathrm{dec}}$ so, for $t<t_{\mathrm{dec}}$, they must be treated as a single component, and one often writes

$\rho_{\mathrm{b} \gamma}=\rho_{\mathrm{b}}+\rho_{\gamma}$.

Irrespective of which components may be dominant or interacting, however, the perturbed Einstein equations feature a single stressenergy tensor representing the total energy density and pressure. In the standard model, one must therefore make additional simplifying assumptions concerning some of the components. One typically adopts the view that only one (or at most two) components dominate the energy density and that the non-interacting components affect the cosmic fluid only gravitationally. Of course, a perfect fluid description can be applied to these components only so long as their mean free paths are shorter than the scales of interest. For example, after decoupling, photons stream freely and form a homogeneous distribution that we can approximate as a 'smooth' background. The data also suggest that neutrino masses are probably small enough to have only a minimal impact on structure formation, so neutrinos too are usually approximated as a smooth radiation component.

These issues apply with equal validity to $R_{\mathrm{h}}=c t$, except for one principal difference. In order for the cosmic fluid to maintain a fixed equation of state $w \equiv p / \rho=-1 / 3$, dark energy cannot be a cosmological constant (Melia 2015a; Melia \& Fatuzzo 2016); it must be dynamic, possibly with a particulate origin in physics beyond the standard model. If we now follow a prescription similar to that outlined in equations (20)-(23), then the simplest assumption we can make (Melia \& Fatuzzo 2016) is that dark energy and radiation (with a contribution from both photons and neutrinos) dominated at early times (i.e. $\rho \approx \rho_{\mathrm{de}}+\rho_{\mathrm{r}}$ for $z \gg 1$ ), while dark energy and matter dominate our local universe (i.e. $\rho \approx \rho_{\mathrm{de}}+\rho_{\mathrm{m}}$ for small $z$ ). One can easily show (Melia \& Fatuzzo 2016) that for $z \gg 1$, this prescription would be consistent with the partitioning

$\rho_{\mathrm{de}} \approx \frac{2}{1-3 w_{\mathrm{de}}} \rho_{\mathrm{c}}(1+z)^{2} \quad(z \gg 1)$,

and

$\rho_{\mathrm{r}} \approx \frac{3 w_{\mathrm{de}}+1}{3 w_{\mathrm{de}}-1} \rho_{\mathrm{c}}(1+z)^{2} \quad(z \gg 1)$,

where $\rho_{\mathrm{c}} \equiv 3 H_{0}(0)^{2} / 8 \pi G$ is the critical density. Thus, if $w_{\mathrm{de}}$ were $-1 / 2$ towards higher redshifts, we would have $\rho_{\mathrm{de}} \approx 0.8 \rho$ and $\rho_{\mathrm{r}} \approx$ $0.2 \rho$. In this (perhaps oversimplified) scheme, one infers a gradual evolution in the relative abundance of the various components, suggesting that they remain coupled at all times. For $z \rightarrow 0$, radiation would no longer have been dynamically important and $\rho$ would have been dominated by $\rho_{\mathrm{m}}$ and $\rho_{\mathrm{de}}$. Again, it is straightforward to show (Melia \& Fatuzzo 2016) that, in order to maintain zero active mass (i.e. $\rho+3 p=0$ ),

$\frac{\rho_{\mathrm{m}}}{\rho} \approx 1+\frac{1}{3 w_{\mathrm{de}}} \quad(z<15)$

For example, if $w_{\mathrm{de}}=-1 / 2$, this suggests that $\rho_{\mathrm{m}} / \rho \approx 1 / 3$ at low redshifts.

Quantum fluctuations emerging out of the Planck regime (Melia 2016b) would have seeded perturbations in this background fluid.
A primordial scalar field $\phi$ with zero active mass, i.e. with an equation of state $\rho_{\phi}+3 p_{\phi}=0$, where $\rho_{\phi}$ and $p_{\phi}$ are its energy density and pressure, respectively, would have produced an essentially scale-free fluctuation spectrum without inflation. This mechanism is based on the Hollands-Wald concept of a minimum wavelength for the emergence of quantum fluctuations into the semiclassical universe (Hollands \& Wald 2002). In this scenario, the $1^{\circ}-10^{\circ}$ fluctuations in the cosmic microwave background (CMB) correspond almost exactly to the Planck length at the time these modes were produced. In contrast to the situation in $\Lambda \mathrm{CDM}$, where the fluctuations transition back and forth across the gravitational horizon, the fluctuations in $R_{\mathrm{h}}=c t$ have a wavelength that grows in proportion to $R_{\mathrm{h}}$, and therefore the perturbations in this cosmology grow in amplitude while their wavelength remains a fixed fraction of the Hubble radius.

Current observations suggest that dark energy forms a smooth background and does not condense. Thus, except in the late stages of clumping, when matter would have collapsed completely to form stars and galaxies, most of the perturbation growth would have been driven by quantum fluctuations $\delta \rho=\delta \rho_{\phi}$ that transitioned first into $\delta \rho \sim \delta \rho_{\mathrm{b} \gamma}$ (dominated by radiation, though with a 'contamination' of baryonic matter) in the early universe, followed by another transition into $\delta \rho \sim \delta \rho_{\mathrm{m}}$ (dominated by matter) at later times.

This is the framework we shall assume in using equation (18) to derive the growth equation for matter perturbations when radiation is relatively unimportant, i.e. when $\rho \approx \rho_{\mathrm{m}}+\rho_{\text {de }}$. Since in this case $v_{\mathrm{s}} \approx 0$ for matter, we have

$\ddot{\tilde{\delta}}_{k}+\frac{3}{t} \dot{\tilde{\delta}}_{k}-\frac{A}{t^{2}} \tilde{\delta}_{k}=0$,

whose solution is the simple polynomial

$\tilde{\delta}_{k}(t)=\left(C_{1} t^{-2}+C_{2} t^{A / 2}\right)$,

where the coefficients $C_{1}$ and $C_{2}$ depend on initial conditions. Ignoring the inconsequential decaying mode, we therefore conclude that

$\tilde{\delta}_{k}(t) \approx \tilde{\delta}_{k}\left(t_{0}\right)\left(\frac{t}{t_{0}}\right)^{A / 2}$,

where the quantity $\tilde{\delta}_{k}\left(t_{0}\right)$ is the k-mode amplitude of the fluctuation today, i.e. at time $t_{0}$. And since $1+z=t_{0} / t$, we may also write equation (29) as

$\tilde{\delta}_{k}(z) \approx \tilde{\delta}_{k}(0)(1+z)^{-A / 2}$.

\subsection{Variance of the perturbations}

The perturbation $\tilde{\delta}_{k}(t)$ is usually assumed to be a Gaussian random field, which means that the waves in the decomposition of equation (16) have random phases. In this instance, the field may be specified entirely by its power spectrum

$$
\begin{aligned}
P(\boldsymbol{k}) & =\left\langle\tilde{\delta}_{k}^{*} \tilde{\delta}_{k}\right\rangle \\
& =\left\langle\left|\tilde{\delta}_{k}\right|^{2}\right\rangle .
\end{aligned}
$$

And for an isotropic distribution, the power spectrum, averaged over all possible realizations, must be independent of direction:

$P(k)=\frac{1}{4 \pi} \oint\left\langle\left|\tilde{\delta}_{k}\right|^{2}\right\rangle \mathrm{d} \Omega$. 
The power spectrum may also be written as the Fourier transform of the autocorrelation function using the Wiener-Khinchin theorem:

$$
\begin{aligned}
\left\langle\delta^{*}(\boldsymbol{x}) \delta(\boldsymbol{x}+\boldsymbol{y})\right\rangle & =\left\langle\int \frac{\mathrm{d}^{3} k^{\prime}}{(2 \pi)^{3}} \int \frac{\mathrm{d}^{3} k}{(2 \pi)^{3}} \tilde{\delta}_{k^{\prime}}^{*} \tilde{\delta}_{k} \times \mathrm{e}^{\mathrm{i} \boldsymbol{k}^{\prime} \cdot \boldsymbol{x}} \mathrm{e}^{-\mathrm{i} \boldsymbol{k} \cdot(\boldsymbol{x}+\boldsymbol{y})}\right\rangle \\
& =\int \frac{\mathrm{d}^{3} k}{(2 \pi)^{3}} P(\boldsymbol{k}) \mathrm{e}^{-\mathrm{i} \boldsymbol{k} \cdot \boldsymbol{y}},
\end{aligned}
$$

whose inversion gives

$P(\boldsymbol{k})=\int \mathrm{d} \boldsymbol{y}\left\langle\delta^{*}(\boldsymbol{x}) \delta(\boldsymbol{x}+\boldsymbol{y})\right\rangle \mathrm{e}^{\mathrm{i} \boldsymbol{k} \cdot \boldsymbol{y}}$.

Using $\boldsymbol{y}$ as the axis about which the poloidal and azimuthal angles are integrated to reduce the general expression to a single integral over $k$, one may write equation (33) more conveniently as

$\left\langle\delta^{*}(\boldsymbol{x}) \delta(\boldsymbol{x}+\boldsymbol{y})\right\rangle=4 \pi \int \frac{k^{2} \mathrm{~d} k}{(2 \pi)^{3}} P(k) \frac{\sin k y}{k y}$,

where $y=|\boldsymbol{y}|$. The variance $\sigma$ of $\delta\left(x^{\alpha}\right)$ is given by the autocorrelation function at $\boldsymbol{y}=0$ :

$\sigma^{2}=4 \pi \int \frac{k^{2} \mathrm{~d} k}{(2 \pi)^{3}} P(k)$.

However, since the fluctuations $\delta(\boldsymbol{x})$ exist on all spatial scales, a more practical measure to use when comparing the power spectrum to the data is the variance delimited within a specified volume. For this purpose, a window function $W_{R}(\boldsymbol{x})$ is introduced with a characteristic radius $R$, such that $W_{R}$ is non-zero for $|\boldsymbol{x}|<R$ and decreases to zero for $|\boldsymbol{x}| \gg R$. The perturbation is then replaced by the convolution integral

$\delta_{R}\left(x^{\alpha}\right) \equiv \int \delta(\boldsymbol{y}, t) W_{R}(|\boldsymbol{x}-\boldsymbol{y}|) \mathrm{d}^{3} y$

and, correspondingly, the power spectrum $P(k)$ must be replaced with $P(k) \tilde{W}_{R}^{2}(k)$, where $\tilde{W}_{R}$ is the Fourier transform of $W_{R}(\boldsymbol{x})$. The volume-delimited variance may thus be written

$\sigma_{R}^{2}=4 \pi \int \frac{k^{2} \mathrm{~d} k}{(2 \pi)^{3}} P(k) \tilde{W}_{R}^{2}(k)$.

For a conventional Gaussian window,

$W_{R}(y)=\frac{1}{(2 \pi)^{3 / 2} R^{3}} \mathrm{e}^{-y^{2} / 2 R^{2}}$,

the Fourier transform is

$\tilde{W}_{R}(k)=\mathrm{e}^{-(k R)^{2} / 2}$,

so putting together equations (31) and (38)- (40), we arrive at the final expression for $\sigma_{R}$ in the $R_{\mathrm{h}}=c t$ universe:

$\sigma_{R}^{2}(z)=4 \pi \int \frac{k^{2} \mathrm{~d} k}{(2 \pi)^{3}}\left\langle\left|\tilde{\delta}_{k}(z)\right|^{2}\right\rangle \mathrm{e}^{-(k R)^{2}}$.

By convention, this variance is usually calculated in spherical volumes with a radius of $8 h^{-1} \mathrm{Mpc}$. Thus, defining

$\sigma_{R}^{2}(0) \equiv 4 \pi \int \frac{k^{2} \mathrm{~d} k}{(2 \pi)^{3}}\left\langle\left|\tilde{\delta}_{k}(0)\right|^{2}\right\rangle \mathrm{e}^{-(k R)^{2}}$,

the volume-delimited variance of $\delta\left(x^{\alpha}\right)$ in the $R_{\mathrm{h}}=c t$ universe is given by the simple expression:

$\sigma_{8}^{R_{\mathrm{h}}=c t}(z) \approx \sigma_{8}^{R_{\mathrm{h}}=c t}(0)(1+z)^{-A / 2}$,

and since $|A| \ll 1$, we have for the low-redshift limit

$\sigma_{8}^{R_{\mathrm{h}}=c t}(z) \approx \sigma_{8}^{R_{\mathrm{h}}=c t}(0)$.
This expression is valid as long as $v_{\mathrm{s}} \approx 0$, i.e. as long as $\delta$ is primarily a fluctuation of matter decoupled (other than through gravity) from the smooth dark energy background. It breaks down when $\rho$ includes a non-negligible contribution from radiation.

\subsection{Observables in the $R_{\mathrm{h}}=c t$ universe}

Measuring the growth rate of cosmological density perturbations is a promising method of testing cosmological models, given that $\tilde{\delta}_{k}(z)$ may in some cases depend sensitively on the underlying expansion rate $H(z)$ and, therefore, on the equation of state $p=w \rho$. The evolution in the variance $\sigma_{8}^{R_{\mathrm{h}}=c t}(z)$ is a manifestation of this growth rate, but galaxies form only in the densest regions of the Universe, so their observed distribution $\delta_{\mathrm{g}}$ is related to the matter density perturbations $\delta$ via a non-trivial bias factor $b$ : $\delta_{\mathrm{g}}=b \delta$. Unfortunately, this bias varies between different populations of galaxies, so measurements of $\sigma_{8}$ from different surveys are difficult to combine and compare with theoretical predictions.

An alternative approach is based on the measurement of peculiar velocities from RSD in a galaxy redshift survey, as first proposed by Kaiser (1987). These peculiar velocities represent (small) deviations from a pure Hubble flow, and are proportional to the so-called cosmological growth factor

$f(a) \equiv \frac{\mathrm{d} \ln D(a)}{\mathrm{d} \ln a}$,

where $D(a)$ represents the growth of matter fluctuations, defined by the expression

$\tilde{\delta}_{k}(a)=\tilde{\delta}_{k}(1) D(a)$.

In the $R_{\mathrm{h}}=c t$ universe, $a(t)=t / t_{0}$, so

$D^{R_{\mathrm{h}}=c t}(a)=a^{A / 2}$,

which means that, in this cosmology, $f^{R_{\mathrm{h}}=c t}(a)$ or, equivalently, $f^{R_{\mathrm{h}}=c t}(z)$, is constant in the same redshift range where $\sigma_{8}$ is a very weak function of $z$.

Over the past several decades, $f(z)$ has been measured using a range of techniques and surveys, including 2dFGRS (Peacock et al. 2001), VVDS (Guzzo et al. 2008), quasar clustering and Ly $\alpha$ clustering (Viel et al. 2004; Ross et al. 2007; da Angela et al. 2008), and in peculiar velocity surveys at $z \sim 0$ (Davis et al. 2011; Hudson $\&$ Turnbull 2012). For example, growth rate measurements may be made using the galaxy two-point correlation function, which yields a value of the parameter $\beta \equiv f / b$. Thus, here too the measured value of $f(z)$ is known from the galaxy distribution only to within a bias factor $b$. Clearly, the probative power of both $\sigma_{8}(z)$ and $f(z)$ is mitigated by the uncertainty in this inferred bias. But each has a dependence on $b$ that is the inverse of the other, so the product $f(z) \sigma_{8}(z)$ may be a much more suitable measure of the structural evolution (Percival \& White 2009; Macaulay et al. 2013; Pavlov, Farooq \& Ratra 2014; Alam et al. 2016). Measurements of the growth rate are now commonly reported using the quantity $f(z) \sigma_{8}(z)$, and these are the data we will employ for the analysis in this paper (see Table 2).

Care must be taken with the use of these data, however, because the measurements are not all completely independent (Alam et al. 2016). Though based on six different (mostly independent) surveys, in some cases probing different biased tracers, they are none the less essentially diagnosing the same matter density field, so some of the sampled volumes overlap. Alam et al. (2016) have calculated the fractional overlap volume between each pair of samples, from which they then estimated the correlation between the corresponding measurements (see their fig. 2). 
Table 2. Measurement of $f \sigma_{8}(z)$ from various redshift surveys.

\begin{tabular}{|c|c|c|c|}
\hline$z$ & $f(z) \sigma_{8}(z)$ & Survey & Reference \\
\hline 0.067 & $0.42 \pm 0.05$ & 6dFGRS(2012) & $\begin{array}{c}\text { (Jones et al. 2009; Beutler } \\
\text { et al. 2012) }\end{array}$ \\
\hline 0.22 & $0.42 \pm 0.07$ & WiggleZ(2011) & (Blake et al. 2011) \\
\hline 0.25 & $0.35 \pm 0.06$ & SDSS LRG(2011) & (Eisenstein et al. 2011) \\
\hline 0.37 & $0.46 \pm 0.04$ & SDSS LRG(2011) & (Eisenstein et al. 2011) \\
\hline 0.41 & $0.45 \pm 0.04$ & WiggleZ(2011) & (Blake et al. 2011) \\
\hline 0.57 & $0.462 \pm 0.041$ & BOSS CMASS & $\begin{array}{c}\text { (Dawson et al. } 2013 \text { Alam } \\
\text { et al. 2015) }\end{array}$ \\
\hline 0.60 & $0.43 \pm 0.04$ & WiggleZ(2011) & (Blake et al. 2011) \\
\hline 0.78 & $0.38 \pm 0.04$ & WiggleZ(2011) & (Blake et al. 2011) \\
\hline 0.80 & $0.47 \pm 0.08$ & Vipers(2013) & (de la Torre et al. 2013) \\
\hline
\end{tabular}

Table 3. Observed values of $f \sigma_{8}(z)$ recalibrated using equation (51) for the $R_{\mathrm{h}}=c t$ cosmology

\begin{tabular}{|c|c|c|c|}
\hline$z$ & $f(z) \sigma_{8}(z)$ & Survey & Reference \\
\hline 0.067 & $0.41 \pm 0.05$ & 6dFGRS(2012) & $\begin{array}{c}\text { (Jones et al. 2009; Beutler } \\
\text { et al. 2012) }\end{array}$ \\
\hline 0.22 & $0.40 \pm 0.07$ & WiggleZ(2011) & (Blake et al. 2011) \\
\hline 0.25 & $0.33 \pm 0.06$ & SDSS LRG(2011) & (Eisenstein et al. 2011) \\
\hline 0.37 & $0.43 \pm 0.04$ & SDSS LRG(2011) & (Eisenstein et al. 2011) \\
\hline 0.41 & $0.42 \pm 0.04$ & WiggleZ(2011) & (Blake et al. 2011) \\
\hline 0.57 & $0.44 \pm 0.04$ & BOSS CMASS & $\begin{array}{c}\text { (Dawson et al. 2013; Alam } \\
\text { et al. 2015) }\end{array}$ \\
\hline 0.60 & $0.40 \pm 0.04$ & WiggleZ(2011) & (Blake et al. 2011) \\
\hline 0.78 & $0.36 \pm 0.04$ & WiggleZ(2011) & (Blake et al. 2011) \\
\hline 0.80 & $0.44 \pm 0.08$ & Vipers(2013) & (de la Torre et al. 2013) \\
\hline
\end{tabular}

For our model comparisons, which are based exclusively on the data listed in Table 2 (and, correspondingly, the data in Table 3 recalibrated for $R_{\mathrm{h}}=c t$ ), we use maximum likelihood estimation with a likelihood function

$\mathcal{L} \propto \mathrm{e}^{-\chi^{2} / 2}$

where

$\chi^{2}=\Delta^{T} C^{-1} \Delta$.

Here, $C^{-1}$ is the inverse of the covariance matrix calculated from the measurement errors quoted in Table 2 and the correlation matrix, and $\Delta$ is the column vector expressing the differences between the measured and predicted values of $f \sigma_{8}$. Its individual components are

$\left.\Delta_{i} \equiv f \sigma_{8}\left(z_{i}\right)\right|_{\text {theory }}-\left.f \sigma_{8}\left(z_{i}\right)\right|_{\text {obs }}$.

But before we can test the model, we must address an additional complication. The galaxy surveys do not measure distances directly. To convert from a measured redshift to a physical distance, one must pre-assume a cosmological model. The problem, of course, is that additional (artificial) anisotropies due to the Alcock-Paczyński effect (Alcock \& Paczyński 1979; Melia \& López-Corredoira 2016) may be imposed on the RSD if the chosen model is incorrect. One must therefore recalibrate the data for each model being tested. All of the entries in Table 2, with the exception of the BOSS datum, were obtained assuming a fiducial $\Lambda \mathrm{CDM}$ cosmology with WMAP optimized parameter values (Hinshaw et al. 2013). The BOSS measurement was made with the Planck concordance model as background (Planck Collaboration XV 2014).

Fortunately, there is a way of transforming the three-dimensional two-point correlation function from one model to another using the Alcock-Paczyński effect (Macaulay et al. 2013). Using 'fid' to designate parameter values optimized with the pre-assumed fiducial model, one may obtain the corresponding quantities in the model being tested using the approximate formula

$\left[f \sigma_{8}(z)\right]^{R_{\mathrm{h}}=c t}=\left[f \sigma_{8}(z)\right]^{\mathrm{fid}} \times B$,

where

$B \equiv \frac{H^{\mathrm{fid}}(z)}{H^{R_{\mathrm{h}}=c t}(z)} \frac{d_{A}^{\mathrm{fid}}(z)}{d_{A}^{R_{\mathrm{h}}=c t}(z)}$.

In this expression, $H(z)$ is the redshift-dependent Hubble constant, and $d_{A}$ is the angular diameter distance. For the application we are considering in this paper, we have

$H^{R_{\mathrm{h}}=c t}(z)=H(0)(1+z)$,

$H^{\mathrm{fid}}(z)=H(0)\left[\Omega_{\mathrm{m}}(1+z)^{3}+\left(1-\Omega_{\mathrm{m}}-\Omega_{\Lambda}\right)(1+z)^{2}+\Omega_{\Lambda}\right]^{1 / 2}$,

and

$d_{A}^{R_{\mathrm{h}}=c t}(z)=\frac{c}{H(0)} \frac{1}{(1+z)} \ln (1+z)$,

$d_{A}^{\mathrm{fid}}(z)=\frac{c}{H(0)} \frac{1}{(1+z)} \int_{0}^{z} \mathrm{~d} u \frac{H(0)}{H(u)}$.

Note that we are here using the symbol $H(0)$ to represent the Hubble constant today in order to avoid confusion with our previously defined $H_{0}$, while $\Omega_{i} \equiv \rho_{i} / \rho_{\mathrm{c}}$ is the current fractional energy density of species ' $i$ ' in terms of the critical density $\rho_{\mathrm{c}} \equiv 3 c^{2} H_{0}^{2} / 8 \pi G$. The measured values of $\left[f \sigma_{8}(z)\right]^{R_{\mathrm{h}}=c t}$ for the $R_{\mathrm{h}}=c t$ cosmology, recalibrated using this procedure, are listed in Table 3.

\section{PERTURBATION GROWTH IN $\Lambda$ CDM}

The linear growth equation in $\Lambda \mathrm{CDM}$, which may also be derived from the formalism in Section 2, is well known and we simply adopt the key results from previous work (e.g. Linder 2005). It is often written in the form

$\ddot{\delta}_{k}+2 H \dot{\delta}_{k}=\frac{4 \pi G}{c^{2}} \rho \delta_{k}$,

where, as usual, dot indicates a derivative with respect to cosmic time. The Hubble constant $H(z)$ is defined in equation (54) and, in the case of $\Lambda \mathrm{CDM}, \Omega_{\Lambda}$ is assumed to be a cosmological constant (for which the dark energy pressure is $p_{\mathrm{de}}=-\rho_{\Lambda}$ ). In addition, for flat $\Lambda \mathrm{CDM}$ (the fiducial model used here), $\Omega_{k}=0$. We have adopted the Planck optimized values (Planck Collaboration XVI 2014), for which $H(0)=67.4 \pm 1.4 \mathrm{~km} \mathrm{~s}^{-1} \mathrm{Mpc}^{-1}, \Omega_{\mathrm{m}}=0.314 \pm 0.020$, and $\Omega_{\Lambda}=0.686 \pm 0.020$.

The solution to equation (57) may be written

$\delta_{k} \propto D^{\Lambda \mathrm{CDM}}(z)=D_{0} H^{\mathrm{fid}}(z) \int_{z}^{\infty} \frac{1+z^{\prime}}{H^{\mathrm{fid}}\left(z^{\prime}\right)^{3}} \mathrm{~d} z^{\prime}$,

where $D_{0}$ is a normalization constant. Therefore,

$\sigma_{8}^{\Lambda \mathrm{CDM}}(z)=\sigma_{8}^{\Lambda \mathrm{CDM}}(0) D^{\Lambda \mathrm{CDM}}(z)$,

normalized such that $D^{\Lambda \mathrm{CDM}}(0)=1$. In addition, we may calculate the growth factor in this model using

$f^{\Lambda \mathrm{CDM}}(z)=\frac{\ddot{a} a}{\dot{a}^{2}}-1+\frac{5 \Omega_{\mathrm{m}}}{2} \frac{(1+z)^{2} H_{0}^{2}}{H^{\mathrm{fid}}(z)^{2} D^{\Lambda \mathrm{CDM}}(z)}$. 


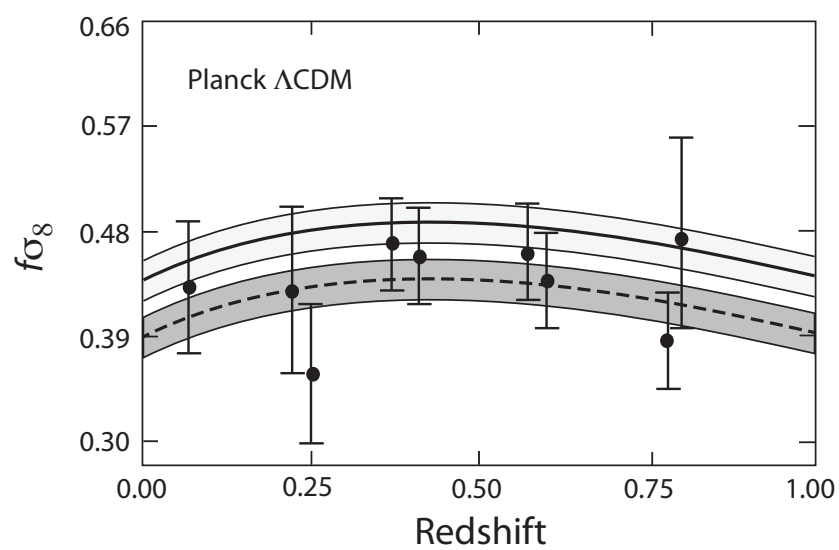

Figure 1. Measured values of $f \sigma_{8}(z)$ versus redshift from various surveys, as indicated in Table 2. The data plotted here have all been recalibrated for the Planck cosmology (Planck Collaboration XVI 2014). The solid curve shows the Planck $\Lambda$ CDM prediction, based on the joint analysis of the Planck measurements and the growth rate measurements listed in Table 2, with the shaded (light grey) band giving the $1 \sigma$ confidence region (adapted from Alam et al. 2016). The $\chi^{2}$ for this fit is 8.4 with $9-3=6$ degrees of freedom. The dashed curve, and its corresponding $1 \sigma$ (dark grey) confidence region, shows the $\Lambda \mathrm{CDM}$ fit based solely on the linear growth data in Table 2 . The $\chi^{2}$ for this fit is 4.03 with $9-1=8$ degrees of freedom (though with the adoption of two priors).

The clumping of matter in $\Lambda \mathrm{CDM}$ is being studied at both high redshifts, primarily with the analysis of anisotropies in the CMB (see e.g. Planck Collaboration XV 2014; Planck Collaboration XVI 2014), and at low redshifts, with weak lensing, galaxy clustering, and the abundance of galaxy clusters (see e.g. MacCrann et al. 2015). The current status of this work points to some tension between the predictions of $\Lambda \mathrm{CDM}$ and the measured linear growth rate at low and high redshifts. The primary CMB anisotropies place limits on the matter fluctuation amplitude at the time of recombination that may be extrapolated to the nearby universe. But the low-redshift measurements seem to be finding a lower value for this fluctuation amplitude than is expected in $\Lambda \mathrm{CDM}$ (Vikhlinin et al. 2009; Beutler et al. 2014; Planck Collaboration XV 2014; Planck Collaboration XVI 2014; MacCrann et al. 2015).

We can see this effect directly by comparing the two theoretical curves superimposed on the fiducial data in Fig. 1. The solid curve, with its associated (light grey) $1 \sigma$ confidence region, shows the optimized $\Lambda \mathrm{CDM}$ fit based on the joint analysis of the Planck and linear growth rate data (adapted from Alam et al. 2016). The $\chi^{2}$ for this fit is 8.4 , with $9-3=6$ degrees of freedom. By comparison, the dashed curve (and associated dark grey $1 \sigma$ confidence region) shows $\Lambda$ CDM's best fit based solely on the growth rate data in Table 2 . The $\chi^{2}$ for this fit is 4.03 with $9-1=8$ degrees of freedom. The principal difference between these two curves is the value of $\sigma_{8}(0)$. The latter optimization has a local fluctuation amplitude approximately 10 per cent smaller than that of the former, which is dominated by the value of $\sigma_{8}(z)$ at recombination. We will rejoin this discussion shortly, following our analysis of $f \sigma_{8}(z)$ for the $R_{\mathrm{h}}=$ ct cosmology.

\section{DISCUSSION}

The data recalibrated using equation (51) for the $R_{\mathrm{h}}=c t$ cosmology are shown in Fig. 2, together with the optimized theoretical fit in this model. For the purpose of this analysis, $\sigma_{8}(0)$ is the sole free

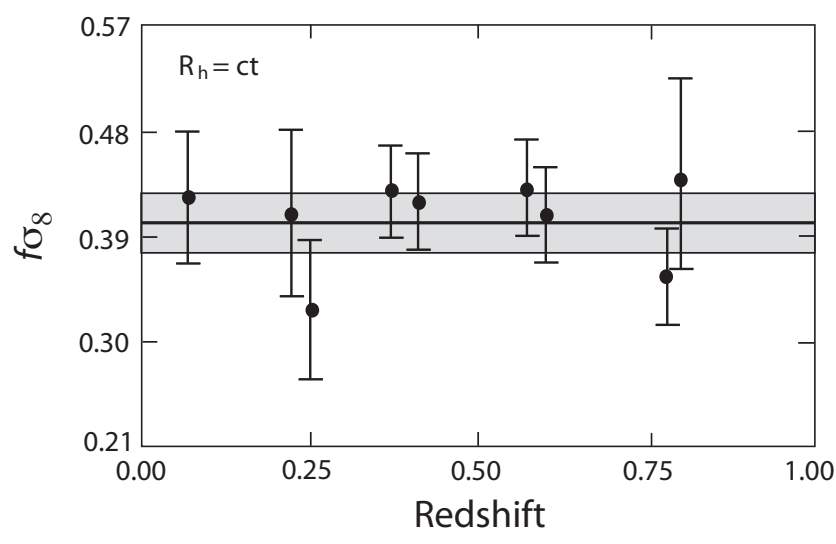

Figure 2. Same as Fig. 1, except the data here have been recalibrated using equation (51) for the $R_{\mathrm{h}}=c t$ universe, as indicated in Table 3 . The solid curve shows the best fit in this cosmology, and the shaded region is the $1 \sigma$ confidence region, corresponding to the optimized value $f \sigma_{8}(0)=0.40 \pm$ 0.03. The $\chi^{2}$ for this fit is 4.3 , with $9-1=8$ degrees of freedom.

parameter that may be adjusted in the fitting procedure. Also shown in Fig. 2 is the $1 \sigma$ confidence region, corresponding to the bestfitting value $f \sigma_{8}(0)=0.40 \pm 0.03$, with a total $\chi^{2}$ of 4.8 and $9-1=8$ degrees of freedom. Clearly, the $R_{\mathrm{h}}=c t$ cosmology fits the linear growth rate data very well, arguably even better than the version of $\Lambda \mathrm{CDM}$ optimized to fit both the Planck and growth rate data (Fig. 1, solid curve; adapted from Alam et al. 2016). We stress, however, that when $\triangle \mathrm{CDM}$ is optimized to fit solely the linear growth rate data (Table 2; Fig. 1, dashed curve), the quality of the $R_{\mathrm{h}}=c t$ fit is statistically indistinguishable from that of $\Lambda \mathrm{CDM}$ when two of its free parameters are assumed to have prior values.

In future work, it will be essential to examine how the growth rate in $R_{\mathrm{h}}=c t$ impacts our interpretation of the CMB anisotropies, particularly with regard to the implied value of $\sigma_{8}^{R_{\mathrm{h}}=c t}(z)$ at recombination, and a comparison of its extrapolated value with $\sigma_{8}^{R_{\mathrm{h}}=c t}(0)$ measured locally. Unfortunately, the value of $\sigma_{8}^{\Lambda \mathrm{CDM}}(0)$ optimized for $\Lambda \mathrm{CDM}$ does not apply to $R_{\mathrm{h}}=c t$, whose angular diameter distance and the ratio $\rho_{\mathrm{m}} / \rho$ at high redshift are quite different from their counterparts in $\Lambda$ CDM (see e.g. Melia \& Shevchuk 2012; Melia \& Fatuzzo 2016). The value of $\sigma_{8}^{R_{\mathrm{h}}=c t}(0)$ presented in this paper is in fact the first (and, so far, only) evaluation of this fluctuation amplitude in the context of $R_{\mathrm{h}}=c t$.

Given these limitations, and fully acknowledging this important caveat, we will for now directly compare the growth rate in $R_{\mathrm{h}}=$ ct measured locally with that implied by the version of $\Lambda \mathrm{CDM}$ optimized to fit both the low- and high-redshift data. It is quite evident that the quality of the fit is not the only indication that $R_{\mathrm{h}}=c t$ may be preferred by these data. Even a simple inspection by eye would suggest that these measurements, at least as shown in Fig. 2, point to an absence of significant curvature in the measured functional dependence of $f \sigma_{8}(z)$ on redshift. This is borne out by Fig. 3, which compares the residuals in $\Lambda \mathrm{CDM}$ with those in $R_{\mathrm{h}}=$ $c t$. Note, in particular that, whereas six out of the nine measurements in $R_{\mathrm{h}}=c t$ lie within $1 \sigma$ of the best-fitting curve, only four do so in $\Lambda$ CDM. Worse, all five of the remaining points in the standard model lie below the best-fitting curve, while a purely randomized distribution should have been evenly dispersed above and below it. At the moment, this asymmetry in the $\Lambda \mathrm{CDM}$ residuals is an even more compelling argument in favour of $R_{\mathrm{h}}=c t$ than a simple comparison of their $\chi^{2}$ values. 


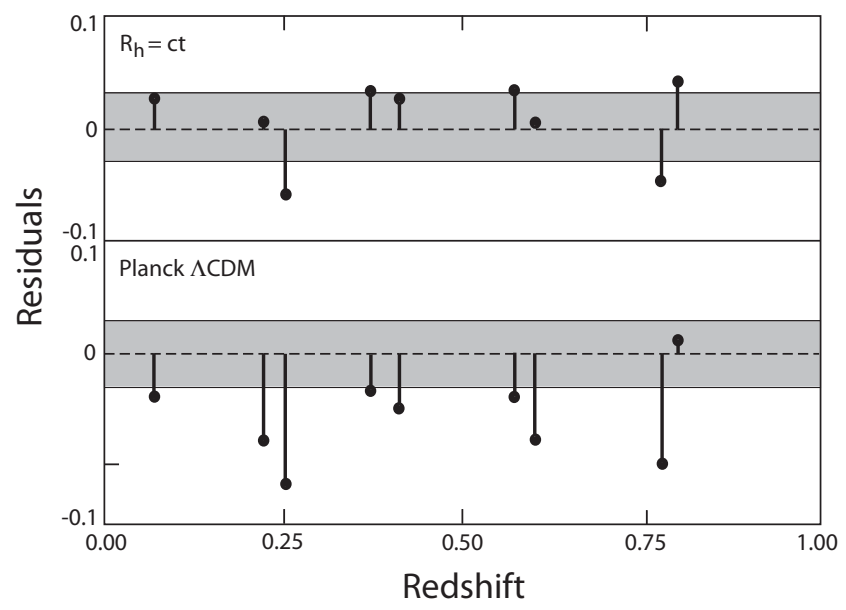

Figure 3. Residuals relative to the best-fitting (solid) curves in Figs 1 and 2 . Shaded sections correspond to $1 \sigma$ confidence regions. For $\Lambda \mathrm{CDM}$, only four of the nine points lie within $1 \sigma$ of the optimized model. More critically, all five of the remaining points lie below it. Together with the indication given by the $\chi^{2}$ values, these results suggest that the linear growth rate measurements favour $R_{\mathrm{h}}=c t$ over Planck $\Lambda \mathrm{CDM}$ (Planck Collaboration XVI 2014).

Unfortunately, one cannot be more definitive than this because, in spite of the evident superiority of $R_{\mathrm{h}}=c t$ over $\Lambda \mathrm{CDM}$ based on their residuals (and, to some degree, on the quality of the fits), the growth rate measurements are not yet accurate enough for us to clearly distinguish a model whose best-fitting curve has significant curvature (Fig. 1) from one that does not (Fig. 2).

\section{CONCLUSION}

In recent years, perturbation theory has matured to the point where the predictions of $\Lambda \mathrm{CDM}$ have been compared extensively to measurements of the growth rate $f \sigma_{8}(z)$, and to other models. The current consensus is that Planck $\Lambda$ CDM (Planck Collaboration XVI 2014) is generally well matched to the data, and that no firm evidence exists for extensions to GR (Alam et al. 2016). Several recent analyses, however, have yielded some tension between the value of $\sigma_{8}(0)$ measured using RSD in galaxy surveys and that inferred by fitting anisotropies in the cosmic microwave radiation (Guzzo et al. 2008; Macaulay et al. 2013).

The principal goal of this paper has been to ascertain whether or not the predictions of the $R_{\mathrm{h}}=c t$ universe are also consistent with the measured growth rate at redshifts $z<2-3$. We have found that the current measurements, though still not sufficiently precise to clearly distinguish between $R_{\mathrm{h}}=c t$ and $\Lambda \mathrm{CDM}$, none the less favour the former over the version of the latter optimized by the joint analysis of Planck and linear growth rate data. The two models are statistically indistinguishable when the optimization of the $\Lambda \mathrm{CDM}$ fit is based solely on the growth rate data in Table 2. Our results also suggest that the present consistency of the standard model with the growth rate data may be an artefact of the relatively large errors associated with these measurements, which cannot yet clearly distinguish between functional forms of $f \sigma_{8}(z)$ with and without significant curvature. This work strongly affirms the need for more precise measurements of the growth rate in that critical redshift range $(0<z<1)$ where differences in the growth function $f \sigma_{8}(z)$ between $\Lambda \mathrm{CDM}$ and $R_{\mathrm{h}}=c t$ are most pronounced.

\section{ACKNOWLEDGEMENTS}

It is a pleasure to acknowledge helpful discussions with Christos Tsagas. I am also very grateful to the anonymous referee for his thoughtful and insightful comments. These have led to a significant improvement in the presentation of the paper. Some of this work was carried out at Purple Mountain Observatory in Nanjing, China, and was partially supported by grant 2012T1J0011 from The Chinese Academy of Sciences Visiting Professorships for Senior International Scientists.

\section{REFERENCES}

Alam S. et al., 2015, ApJS, 219, 12

Alam S., Ho S., Silvestri A., 2016, MNRAS, 456, 3743

Alcock C., Paczyński B., 1979, Nature, 281, 358

Amendola L., 2000, MNRAS, 312, 521

Betoule M. et al., 2014, A\&A, 568, 22

Beutler F. et al., 2012, MNRAS, 423, 3430

Beutler F. et al., 2014, MNRAS, 444, 3501

Bikwa O., Melia F., Shevchuk A. S. H., 2012, MNRAS, 421, 3356

Bilicki M., Seikel M., 2012, MNRAS, 425, 1664

Blake C. et al., 2011, MNRAS, 418, 1707

Capozziello S., Cardone V. F., Troisi A., 2005, Phys. Rev. D, 71, 043503

Carroll S. M., Duvvuri V., Trodden M., Turner M. S., 2004, Phys. Rev. D, 70,043528

Cavanaugh J. E., 2004, Aust. N.Z. J. Stat., 46, 257

Coles P., Lucchin F., 1995, Cosmology: The Origin and Evolution of Cosmic Structure, Wiley, New York

Cuesta A. J. et al., 2016, MNRAS, 457, 1770

da Angela J. et al., 2008, MNRAS, 383, 565

Davis M., Nusser A., Masters K. L., Springob C., Huchra J. P., Lemson G., 2011, MNRAS, 413, 2906

Dawson K. S. et al., 2013, AJ, 145, 10

de la Torre S. et al., 2013, A\&A, 557, A54

Dvali G., Gabadadze G., Porrati M., 2000, Phys. Lett. B, 485208

Eisenstein D. J., Seo H.-J., Sirko E., Spergel D. N., 2007, ApJ, 664, 675

Eisenstein D. J. et al., 2011, AJ, 142, 72

Florides P. S., 1980, Gen. Relativ. Gravit., 12, 563

Font-Ribera A. et al., 2014, J. Cosmol. Astropart. Phys., 5, 27

Guzzo L. et al., 2008, Nature, 451, 541

Hinshaw G. et al., 2013, ApJS, 208, 19

Hollands S., Wald R. M., 2002, Gen. Relativ. Gravit., 34, 2043

Hudson M. J., Turnbull S. J., 2012, ApJ, 751, L30

Jain B., Zhang P., 2007, Phys. Rev. D, 78, 063503

Jones D. H. et al., 2009, MNRAS, 399, 683

Kaiser N., 1987, MNRAS, 227, 1

Kolb E. W., Turner M., 1990, The Early Universe. Addison-Wesley, Reading, MA

Landau L. D., Lifshitz E. M., 1975, The Classical Theory of Fields. Pergamon Press, Oxford

Lewis G. F., 2013, MNRAS, 432, 2324

Liddle A. R., 2007, MNRAS, 377, L74

Liddle A. R. D., Lyth K. A., 2000, Cosmological Inflation and Large-Scale Structure. Cambridge Univ. Press, Cambridge

Linder E. V., 2005, Phys. Rev. D, 72, 043529

López-Corredoira M., 2014, ApJ, 781, 96

López-Corredoira M., Melia F., Lusso E., Risaliti G., 2016, Int. J. Mod. Phys. D, 25, 1650060

Macaulay E., Wehus I. K., Eriksen H. K., 2013, Phys. Rev. Lett., 111, 161301

MacCrann N., Zuntz J., Bridle S., Jain B., Becker M. R., 2015, MNRAS, 451, 2877

Melia F., 2007, MNRAS, 382, 1917

Melia F., 2012, J. Cosmol. Astropart. Phys., 09, 029

Melia F., 2014a, J. Cosmol. Astropart. Phys., 01, 027

Melia F., 2014b, AJ, 147, 120

Melia F., 2015a, Ap\&SS, 356, 393 
Melia F., 2015b, Ap\&SS, 359, 34

Melia F., 2015c, MNRAS, 446, 1191

Melia F., 2016a, Frontiers Phys., 11, 118901

Melia F., 2016b, CQG, in press

Melia F., 2016c, Proc. R. Soc. A, 472, 20150765

Melia F., 2017, Frontiers Phys., 12129802

Melia F., Fatuzzo M., 2016, MNRAS, 456, 3422

Melia F., López-Corredoira M., 2016, Int. J. Mod. Phys. D, preprint (arXiv:1503.05052)

Melia F., McClintock T. M., 2015a, AJ, 150, 119

Melia F., McClintock T. M., 2015b, Proc. R. Soc. A, 471, 20150449

Melia F., Maier R. S., 2013, MNRAS, 432, 2669

Melia F., Shevchuk A. S. H., 2012, MNRAS, 419, 2579

Melia F., Wei J.-J., Wu X., 2015, AJ, 149, 2

Mitra A., 2014, MNRAS, 442, 382

Padmanabhan T., 1993, Structure Formation in the Universe. Cambridge Univ. Press, Cambridge

Padmanabhan N. et al., 2012, MNRAS, 427, 2132

Pavlov A., Farooq O., Ratra B., 2014, Phys. Rev. D, 90, 023006

Peacock J. A., 1999, Cosmological Physics. Cambridge Univ. Press, Cambridge

Peacock J. A. et al., 2001, Nature, 410, 169

Peebles P. J. E., 1980, The Large-scale Structure of the Universe. Princeton Univ. Press, Princeton, NJ

Peebles P. J. E., 1993, Principles of Physical Cosmology. Princeton Univ. Press, Princeton, NJ

Percival W. J., White M., 2009, MNRAS, 393, 297

Planck Collaboration XV, 2014, A\&A, 571, A15
Planck Collaboration XVI, 2014, A\&A, 571, A16

Press W. H., Vishniac E. T., 1980, ApJ, 239, 1

Ross N. et al., 2007, MNRAS, 381, 573

Schwarz G., 1978, Ann. Stat., 6, 461

Shafer D. L., 2015, Phys. Rev. D, 91, 103516

Song Y.-S., Koyama K., 2009, J. Cosmol. Astropart. Phys., 01, 048

Song Y.-S., Percival W. J., 2009, J. Cosmol. Astropart. Phys., 10, 004

Suzuki N. et al., 2012, ApJ, 746, 85

Tsagas C. G., Challinor A., Maartens R., 2008, Phys. Rep., 465, 61

van Oirschot P., Kwan J., Lewis G. F., 2010, MNRAS, 404, 1633

Viel M., Haehnelt M. G., Springel V., 2004, MNRAS, 354, 684

Vikhlinin A. et al., 2009, ApJ, 692, 1060

Wei J.-J., Wu X., Melia F., 2013, ApJ, 772, 43

Wei J.-J., Wu X., Melia F., Wei D.-M., Feng L.-L., 2014a, MNRAS, 439, 3329

Wei J.-J., Wu X., Melia F., 2014b, ApJ, 788, 190

Wei J.-J., Wu X., Melia F., Maier R. S., 2015a, AJ, 149, 102

Wei J.-J., Wu X., Melia F., 2015b, AJ, 149, 165

Wei J.-J., Wu X., Melia F., Wang F.-Y., Yu H., 2015c, AJ, 150, 35

Wei J.-J., Wu X., Melia F., 2015d, MNRAS, 447, 479

Weinberg S., 1972, Gravitation and Cosmology. Wiley, New York

Wetterich C., 1995, A\&A, 301, 321

Yu H., Wang F. Y., 2014, Eur. J. Phys. C, 74, 3090

Zeng H., Melia F., Zhang L., 2016, MNRAS, 462, 3094

This paper has been typeset from a $\mathrm{T}_{\mathrm{E}} \mathrm{X} / \mathrm{L} \mathrm{T} \mathrm{E} \mathrm{X}$ file prepared by the author. 